\title{
A LEGITIMIDADE DO ÍNDICE DE SUSTENTABILIDADE EMPRESARIAL (ISE) FRENTE AOS DEMAIS ÍNDICES B3
}

\author{
The legitimacy of the Corporate Sustainability Index (CSI) in relation \\ other Indexes B3
}

\begin{abstract}
Roberto Francisco de Souza
E-mail: robertofsouzajr@gmail.com

Mestre em Contabilidade pela Universidade Estadual do Oeste do Paraná;

Graduado em Ciências Contábeis pela

Faculdade de Ciências Contábeis e Administração do Vale do Juruena; Professor nos Cursos de Graduação em Ciências Contábeis e Administração do Centro Universitário Maurício de Nassau de

Salvador.

Endereço para contato: Rua dos Maçons, 364, 41810-205, Pituba, Salvador, Bahia, Brasil.

https://orcid.org/0000-0001-9776-9139

Adhmir Renan Voltolini Gomes

E-mail: adhmir.renan@gmail.com

Mestre em Contabilidade pela Universidade Estadual do Oeste do Paraná; Especialista em Administração Financeira, Contábil e Controladoria pelo Inbrape. https://orcid.org/0000-0003-2089-5924

Samuel Lyncon Leandro de Lima

E-mail: samuellynconleandro@gmail.com

Mestre em Contabilidade pela Universidade Estadual do Oeste do Paraná;

Graduado em Ciências Contábeis pela Universidade Estadual de Maringá. https://orcid.org/0000-0003-3956-3575
\end{abstract}

\section{Gracielly Vieira dos Santos}

E-mail: graciellyvieira2017@gmail.com

Graduada em Ciências Contábeis pela Universidade Norte do Paraná; Mestranda em Contabilidade pela Universidade Estadual do Oeste do Paraná; Professora no Curso de Ciências Contábeis e Gestão para Cooperativas no Centro TécnicoEducacional Superior do Oeste Paranaense. https://orcid.org/0000-0003-0928-4188

Delci Grapegia Dal Vesco

E-mail: delcigrape@gmail.com

Doutora em Contabilidade e Administração pela Universidade Regional de Blumenau; Mestra em Contabilidade pela Universidade Federal do Paraná; Professora e Coordenadora no Mestrado em Contabilidade e Professora no Mestrado Profissional em Administração da Universidade Estadual do Oeste do Paraná de Cascavel. https://orcid.org/0000-0002-0818-3142

Artigo recebido em 13 de outubro de 2018. Aceito em 2 de outubro de 2019. 


\section{Resumo}

O trabalho analisa a relação e a influência dos vários índices da Brasil, Bolsa, Balcão no Índice de Sustentabilidade Empresarial (ISE). A amostra foi selecionada por meio de séries e das variações diárias de cada índice entre 2 de janeiro de 2012 e 29 de dezembro de 2016, com 22.284 observações no total. Para analisar o retorno dos índices foram delimitadas as variações diárias de cada um, naquele período. Os resultados demonstraram que a relação do ISE com os demais índices é forte, positiva e estatisticamente significante. Portanto, esses resultados apontam que as empresas buscam se legitimar participando desse Índice, isto é, buscam incorporar cada vez mais estruturas isomórficas que refletem o ambiente institucional. $\mathrm{E}$ assim, a adoção do ISE pode ser apenas mais um rótulo. Com isso, questionase a legitimidade desse Índice, pois, se existe relação com todos os indicadores, basta às empresas se suprimirem às regras dos índices que são pertinentes aos seus interesses. As principais influências positivas no Índice de Sustentabilidade Empresarial foram estabelecidas pelos índices IGCT, IVBX, ICO2, IGCX e UTIL. Já os índices IFNC, IMOB, ICON e IBOV influenciam de forma negativa o ISE. Logo, a legitimidade proporcionada pelo ISE pode ser atrelada à composição dos demais índices.

Palavras-chave: Teoria da legitimidade. Desempenho organizacional. ISE. Sustentabilidade.

\section{Abstract}

The paper analyzes the relationship and influence of the various indexes of Brasil, Bolsa, Balcão (B3) on the Corporate Sustainability Index (ISE). The sample was selected through series and daily variations of each index between $1 / 2 / 2012$ to $29 / 12 / 2016$, with 22,284 observations in total. To analyze the return of the indices, the daily variations of each one in that period were delimited. The results showed that the relationship between the ISE and the other indices is strong, positive and statistically significant. Therefore, these results indicate that companies that seek to legitimize themselves by participating in this Index, that is, seek to incorporate more and more isomorphic structures that reflect the institutional environment. And so, the adoption of ISE may just be another label. Thus, the legitimacy of this Index is questioned, because if there is a relationship with all indicators, it is enough for companies to suppress the index rules that are relevant to their interests. The main positive influences on the Corporate Sustainability Index were established by the IGCT, IVBX, ICO2, IGCX and UTIL indices. The IFNC, IMOB, ICON and IBOV indices negatively influence the ISE. Thus, the legitimacy provided by the ISE can be linked to the composition of the other indices.

Keywords: Theory of legitimacy. Organizational performance. ISE. Sustainability.

\section{INTRODUÇÃO}

O Investimento Socialmente Responsável (ISR) apresentou uma enorme expansão, desde sua criação em 1995 nos EUA, e passou a ser utilizado por grandes corporações. A participação voluntária em iniciativas de sustentabilidade gerava ganhos intangíveis como, por exemplo, a reputação e o compartilhamento de experiências na comunidade empresarial (Macedo, Barbosa, Callegari, Monzoni, \& Simonetti, 2012). Nesse caminho, houve consequências no mercado acionário, com o surgimento de carteiras teóricas compostas apenas por empresas que adotassem determinadas práticas ambientais, como, 
por exemplo, Triple Bottom Line. Assim, essas carteiras são bases representativas dos índices de sustentabilidade nas bolsas de valores (Cristófalo, Akaki, Abe, Morano, \& Miraglia, 2016).

Com iniciativa pioneira na América Latina, o Índice de Sustentabilidade Empresarial (ISE) foi elaborado para propiciar um ambiente de investimento voltado para cumprir as demandas de desenvolvimento sustentável e estimular a responsabilidade ética das corporações (Brasil, Bolsa, Balcão [B3], 2019). Nesse sentido, o ISE representou uma nova concepção ao estabelecer que as empresas se esforcem e cumpram os padrões considerados sustentáveis e corretos, com a finalidade de serem reconhecidas por comporem essa carteira (Favaro \& Rover, 2014).

Nessa mesma corrente surgiram novas carteiras teóricas, segmentadas por empresas que adotassem determinadas práticas de gestão com finalidade de atender aos interesses dos stakeholders. Desse modo, novos índices foram estabelecidos como, por exemplo, índice de carbono eficiente, índice governança corporativa novo mercado, índice imobiliário, entre outros.

Embora existam evidências que a visão da redução da poluição gera um custo para as empresas e prejudica a competividade, também é possível encontrar evidencias que a redução aumenta a eficiência e, por consequência, economiza recursos e aumenta a vantagem com relação ao custo (Hart \& Ahuja, 1996).

Com essas práticas, as empresas podem se beneficiar da redução de custos por meio da eficiência ecológica, pois podem desenvolver mercados ecológicos e obter vantagem competitiva de pioneiro, bem como possuir melhores relações com a comunidade e aprimorar a própria imagem. A importância dessas atividades e aspectos ambientais justifica o valor e a utilidade do controle ambiental em apoio ao desempenho ambiental, visto que este influencia positivamente o desempenho econômico (Henri \& Journeault, 2010). A gestão ambiental pode ser considerada como uma capacidade estratégica para as organizações, pois o desempenho econômico é um reflexo do desempenho ambiental (Deliberal, Tondolo, Camargo, \& Tondolo, 2016).

Conforme Oro, Balbinot, Thomé, e Lavarda (2014), no mundo inteiro os consumidores passaram a considerar não apenas o preço e a qualidade dos produtos, mas também o comportamento social das empresas, fazendo com que estas adotem novos posicionamentos administrativos.

Nesse cenário, indaga-se: qual a relação e influência entre os indicadores da Brasil, Bolsa, Balcão no Índice de Sustentabilidade Empresarial? Assim, objetiva-se estabelecer essa relação e a no ISE. A justificativa deste estudo refere-se à existência de vários índices, mas - ISE é o que mais exige certificações. Além disso, as empresas buscam legitimar suas ações por meio dele. Como contribuição teórica, verificar as relações e influências dos demais índices pode demonstrar a autenticidade do ISE no desempenho de mercado das empresas. 
Este artigo está estruturado em cinco partes, incluindo a introdução. A seguir, apresenta-se a revisão da literatura, a qual contempla aspectos relacionados à teoria da legitimidade, ao ISE e ao desempenho organizacional. Na terceira seção, delineiam-se os procedimentos metodológicos, contemplando a abordagem metodológica e a técnica da pesquisa. Na quarta seção, apresentam-se os resultados da pesquisa. $E$, por fim, na quinta seção, as considerações finais.

\section{REFERENCIAL TEÓRICO}

\subsection{TEORIA DA LEGITIMIDADE}

As organizações buscam a legitimidade por diversos motivos, e partindo desse pressuposto, o empenho exigido dessas organizações nessa busca por legitimidade, frente às dificuldades encontradas nesse contexto, está relacionado aos objetivos para alcançar a legitimação (Suchman, 1995). Ainda, de acordo com a visão do autor, por meio dessa proposição, a legitimidade pode ser considerada um mecanismo que as companhias podem utilizar em busca de alavancar a posição de competitividade, dado que o aumento da estabilidade e o entendimento das atividades organizacionais são fatores que estão relacionados à busca de legitimidade.

Nesse sentido, Parsons (1956) define a organização como um sistema social que, para atingir seu objetivo, realiza a produção de determinado bem, para, de alguma forma, ser adquirido por outro sistema. Dessa forma, o autor propõe que o cumprimento de um objetivo está associado à relação de um sistema com as partes externas interessadas em que ele opera, e cabe ressaltar que essa interação é constituída com a maximização de valor frente aos fatores relacionados a custos e obstáculos decorrentes desse processo. Ainda, de acordo com os autores Meyer e Rowan (1977), as empresas se organizam por meio de um conjunto de regras estabelecidas pela sociedade, condição que evidencia o aumento da complexidade das estruturas organizacionais.

Essas organizações incorporam cada vez mais estruturas isomórficas, refletidas pelo ambiente institucional, afastando-se daquela construída com enfoque na produção e na capacidade técnica, uma vez que, nesse estágio, a flexibilidade da gestão e do controle interno é adotada na busca pela legitimação (Meyer \& Rowan, 1977). Suchman (1995) trabalha a legitimidade em três categorias, definidas como legitimidade pragmática, legitimidade moral e legitimidade cognitiva. Nessa perspectiva, o autor pontua que a legitimidade pragmática está relacionada a aspectos de imediatismo da organização, a legitimidade moral está 
associada a aspectos éticos ou crenças e a legitimidade cognitiva possui uma relação com o reconhecimento da organização.

Essa tratativa das empresas, no tocante à legitimidade, possui um reflexo direto no desempenho social e, consequentemente, na divulgação desse desempenho, dado que a preocupação institucional consiste desde a forma de realizar a evidenciação até o conteúdo a ser evidenciado ao público (Lindblom, 1994). Essa proposição pode ser confirmada com o estudo de O'Donovan (2002), na medida em que investigou a associação entre os relatórios evidenciados pelas organizações à tentativa de obtenção, manutenção e reparação da legitimidade; e sobre esse aspecto os resultados apontaram que a legitimidade possui uma grande relevância a respeito do tratamento das divulgações. Cabe ressaltar que a relevância observada ocorre visando à recepção social e, em decorrência disso, à resposta com relação às informações apresentadas.

Assim, na medida em que as organizações se envolverem em situações negativas, com decorrente impacto desfavorável na sua legitimidade, é necessária a utilização de estratégias para responder à evidenciação de relatórios (Lindblom, 1994). Diante disso, o autor propõe quatro estratégias para as organizações: orientar o público de interesse sobre as devidas alterações no desempenho e atividades institucionais; modificar a percepção dos interessados sobre o desempenho da empresa, sem a necessidade de alterar o desempenho atual da empresa; manipular a percepção dos interessados sobre o desempenho da empresa, apresentando assuntos relevantes, relacionados a atividades de cunho social realizadas ou em desenvolvimento por meio da instituição; e inverter as expectativas externas sobre o desempenho futuro da organização.

Diante disso, a legitimidade pode estar relacionada com alguns atributos, como, por exemplo, com a inovação, divulgação, sustentabilidade, entre outros. Os resultados do estudo de Agustia, Sawarjuwono, \& Dianawati, (2019) evidenciaram que a inovação verde possui um efeito estatisticamente significativo do valor da empresa.

No entanto, existem resultados que demonstram que pode ser uma preocupação secundária em decorrência da relação direta da regulação do setor. Os achados de Hermawan, Aisyah, Gunardi, e Putri (2018) demonstraram que além de o regulador influenciar a divulgação de emissão de carbono, o tamanho da empresa e a lucratividade também são determinantes.

Embora tenham investigado o setor de energia, Furtado, Antonovz, Correa, Silva, e Panhoca (2019) concluíram que a inovação pode ser uma forma de legitimar as ações de responsabilidade social corporativa, porém, está mais focada nas obrigações exigidas pelos órgãos reguladores. 
Nessa perspectiva de necessidade das organizações em buscarem a legitimação, com base na divulgação de relatórios com informações significantes, de impacto positivo na sociedade, emergem os indicadores de sustentabilidade, apresentados a seguir.

\section{2 ÍNDICE DE SUSTENTABILIDADE EMPRESARIAL (ISE)}

O conceito de sustentabilidade representa para o setor empresarial uma nova abordagem nas realizações dos negócios, com o intuito de promover a responsabilidade social e a redução do uso de recursos naturais. Consequentemente, reduzem-se os impactos negativos sobre o meio ambiente, preservando a integridade do Planeta para as futuras gerações, sem descontinuar a função objetivo das organizações, expressa por meio da rentabilidade econômico-financeira do empreendimento (Silva, Rocha, Wienhage, \& Rausch, 2009).

Nesse sentido, surge em meados de 2005 o ISE, uma iniciativa tratada na América Latina com intuito de estimular o cenário de investimento com uma abordagem voltada ao desenvolvimento sustentável, para suprir a demanda da sociedade e promover a ética das organizações. Assim, financiado pela International Finance Corporation (IFC), ligada ao Banco Mundial, o ISE foi estruturado metodologicamente pelo Centro de Estudos em Sustentabilidade (GVCes) da Escola de Administração de Empresas de São Paulo da Fundação Getúlio Vargas (FGV-EAESP, 2017). A gestão técnica e o cálculo do índice são definidos sob a responsabilidade da bolsa de valores $(B 3,2017)$.

O ISE é classificado como uma ferramenta para a análise comparativa do desempenho das companhias listadas na Brasil, Bolsa, Balcão, sob a vertente da sustentabilidade corporativa, fundamentada na eficiência econômica, equilíbrio ambiental, justiça social e governança corporativa. Esse entendimento também engloba as organizações $e$ as categorias envolvidas com a sustentabilidade, distinguindo-as em se tratando de qualidade e nível de comprometimento com o desenvolvimento sustentável. Essa distinção amplia-se com os quesitos relacionados à equidade, transparência e prestação de contas, natureza do produto, além do desempenho organizacional sob as abordagens econômico-financeiras, sociais, ambientais e de mudanças climáticas (B3, 2017).

Nesse sentido, congregar no ISE, de acordo com Sales, Rover, e Ferreira (2018), é relevante para as empresas estarem em posição evidente junto ao mercado. Isso se deve ao fato de que o ISE tem como proposta, segundo Favaro e Rover (2014) e Guimarães, Peixoto, e Carvalho (2017), fomentar um cenário orientado pela busca de um desenvolvimento organizacional sustentável. Isto é, atender às demandas correntes e crescentes da sociedade com relação à responsabilidade acerca da sustentabilidade (Guimarães et al., 2017).

Como missão estabelecida, o ISE busca dar suporte aos investidores na tomada de decisão de investimentos socialmente responsáveis e conduzir as organizações na adoção 
às melhores práticas de sustentabilidade empresarial. $O$ indicador é fundamentado sobre quatro princípios. $O$ primeiro consiste na transparência, no que se refere ao processo do ISE e às respostas das organizações ao questionário (FGV-EAESP, 2017).

Por conseguinte, o segundo é atrelado ao diálogo com as partes interessadas e a sociedade, na perspectiva de fomentar o relacionamento com esses elementos em busca de legitimidade, representatividade e atualidade do ISE como a manifestação de expectativas sobre sustentabilidade organizacional (FGV-EAESP, 2017).

O terceiro enfoque é definido como o aperfeiçoamento contínuo do escopo e do processo, idealizado como a atualização anual do questionário ISE, de maneira que seja embasado em pesquisas acadêmicas e processos em que haja interação com o estado da arte do conhecimento em sustentabilidade com os anseios da sociedade em geral (FGV-EAESP, 2017).

Por fim, o quarto fator é compreendido como a autonomia financeira, metodológica e decisória, para garantir os requisitos materiais, técnicos e de gestão, visando para o ISE à legitimidade e à credibilidade primordial para o cumprimento do propósito e o avanço em direção à missão estabelecida (FGV-EAESP, 2017).

Com isso, é possível verificar a proximidade do ISE com a busca de legitimidade, principalmente no tocante aos aspectos fundamentais do indicador. Outro aspecto relacionado ao índice, tratado como função objetiva da empresa, possui vínculo com o desempenho econômico das organizações, assunto da próxima seção.

\subsection{DESEMPENHO ORGANIZACIONAL}

O desempenho, na visão Sonnentage Frese (2002) possui um enfoque multidimensional. Sob a vertente dos negócios, o desempenho deve ser na perspectiva de Venkatraman e Ramanujam (1986), diferenciado da discussão global sobre a eficácia organizacional. 0 desempenho do negócio é o que reflete a perspectiva de gestão estratégica, caracterizado como um subconjunto do conceito global da eficácia organizacional (Venkatraman \& Ramanujam, 1986). Dessa forma, as organizações precisam de indivíduos responsáveis quanto ao desempenho, em busca do cumprimento de metas, visando entregar os produtos e serviços com qualidade e eficiência, promovendo para a organização uma vantagem competitiva (Sonnentag \& Frese, 2002).

Essa preocupação em buscar indivíduos com o foco no desempenho, segundo Brandão e Guimarães (2001), não é uma abordagem recente das organizações. No entanto, a gestão do desempenho é uma questão que emergiu recentemente, propondo um enfoque alternativo aos aspectos tradicionais, trabalhados na avaliação de desempenho. Além disso, a avaliação consiste na comparação dos resultados realizados, com aqueles esperados, predefinidos no 
planejamento (Brandão \& Guimarães, 2001). Cabe ressaltar que o resultado alcançado pela organização está associado ao comportamento dos indivíduos (Sonnentag \& Frese, 2002).

No desempenho financeiro emerge a concepção estreita de centros de desempenho do negócio com a utilização de indicadores financeiros para refletir o cumprimento dos objetivos econômicos da empresa. Assim, refere-se a essa estrutura conceitual do desempenho financeiro, ou medida de desempenho financeiro multidimensional, um conjunto de medidas financeiras. Esse conjunto pode ser classificado em dimensões apresentadas como: a rentabilidade-retorno sobre o investimento ( $\mathrm{ROI}$ ); o ganho antes dos juros e impostos (EBIT); as margens de lucro bruto, o crescimento da quota de mercado, o crescimento-crescimento de vendas; a eficiência -retorno sobre as vendas (ROS); e o retorno sobre o patrimônio (ROE) (Venkatraman \& Ramanujam, 1986).

Todavia, as análises realizadas, usando uma única medida financeira ou diversas ações relativas a uma única dimensão, podem direcionar a conclusões enganosas. Considerando a respectiva condição, o conceito de desempenho do negócio deve proporcionar uma ênfase em medidas de desempenho operacional, que consistem em parâmetros-chave, conduzindo a uma melhoria no desempenho financeiro (Venkatraman \& Ramanujam, 1986).

Diante disso, é importante considerar que o contexto atual, em que as organizações estão posicionadas, consiste em um nível elevado de competitividade, situação que impõe nos gestores um grau relevante de comprometimento no exercício de suas respectivas funções. Esses requisitos, somados à qualificação desses gestores, possuem um foco direcionado à geração de resultados significativos (Nascimento, Rocha, Kroenke, Silva, \& Hein, 2012).

Evidentemente que mecanismos de fiscalização, como a governança corporativa, que possui influência positiva sobre a transparência nas organizações, pode elevar ainda mais o nível de responsabilidade dos gestores (Lima, 2018). Além disso, conforme Lima, Gomes, Priebe, Dall'Asta, e Mello (2018), a governança corporativa pode influenciar positivamente o preço da ação das empresas.

Contudo, ressalta-se que o desempenho econômico e financeiro pode ser afetado pelos custos das práticas de governança, nesse sentido, o custo de fiscalização também pode afetar o desempenho, e isso pode apresentar problemas de endogeneidade. Desse modo, a dissociação temporal da governança e do desempenho é importante, pois as práticas de gestão são tomadas com antecedência (Gomes, 2018).

Fundamentando nisso, entende-se que o planejamento se configura em um pressuposto essencial no processo de avaliação do desempenho. Essa abordagem é verificada na medida em que avaliar o desempenho de uma organização sem os parâmetros relacionados à definição de metas ou resultados esperados se torna um procedimento difícil. Considera-se, assim, que o sucesso consistente no processo de avaliação do desempenho organizacional, está 
particularmente associado à gestão, sendo utilizada como um mecanismo de acompanhamento e de ação permanente em razão de resultados positivos (Nascimento et al., 2012).

Desse modo, diante do exposto, torna-se, plausível a sistemática formada sobre o desempenho organizacional. Com isso, na próxima seção é apresentado o delineamento metodológico empregado na pesquisa.

\section{METODOLOGIA}

Para cumprir com os objetivos propostos, realizou-se uma pesquisa do tipo documental, pois foram coletados dados secundários (Hair, Babin, Money, \& Samouel, 2005), em outras fontes.

Os índices analisados, neste estudo, estão descritos na Tabela 1. Para analisar o retorno dos índices, foram delimitadas as variações diárias de cada índice no período de 2 de janeiro de 2012 a 29 de dezembro de 2016.

Tabela 1

Índices da Brasil, Bolsa, Balcão

\begin{tabular}{|c|c|c|}
\hline Abreviação & Índice & Objetivo \\
\hline ISE & $\begin{array}{l}\text { Índice de } \\
\text { sustentabilidade } \\
\text { empresarial }\end{array}$ & $\begin{array}{l}\text { O ISE é uma ferramenta para análise comparativa da performance } \\
\text { das empresas listadas na Brasil, Bolsa, Balcão, sob aspecto de } \\
\text { sustentabilidade corporativa. }\end{array}$ \\
\hline IBOV & 17 Índice Bovespa & $\begin{array}{l}\text { Indicar o desempenho médio das cotações dos ativos de maior } \\
\text { negociabilidade e representatividade do mercado de ações brasileiro. }\end{array}$ \\
\hline IBRA & 16 Índice Brasil Amplo & $\begin{array}{l}\text { Indicar o desempenho médio das cotações de todos os ativos } \\
\text { negociados no mercado à vista (lote-padrão) da Brasil, Bolsa, Balcão } \\
\text { que atendam a critérios mínimos de liquidez e presença em pregão, de } \\
\text { forma a oferecer uma visão ampla do mercado acionário. }\end{array}$ \\
\hline IBRX & 15 Índice Brasil 100 & $\begin{array}{l}\text { Indicar o desempenho médio das cotações dos } 100 \text { ativos de maior } \\
\text { negociabilidade e representatividade do mercado de ações brasileiro. }\end{array}$ \\
\hline IBXL & 14 Índice Brasil 50 & $\begin{array}{l}\text { Indicar o desempenho médio das cotações dos } 50 \text { ativos de maior } \\
\text { negociabilidade do mercado de ações brasileiro. }\end{array}$ \\
\hline ICO2 & $\begin{array}{l}13 \text { Índice do carbono } \\
\text { eficiente }\end{array}$ & $\begin{array}{l}\text { Incentivar as empresas emissoras das ações mais negociadas a } \\
\text { aferirem, divulgarem e monitorarem suas emissões de GEE. }\end{array}$ \\
\hline ICON & 12 Índice do consumo & $\begin{array}{l}\text { Indicar o desempenho médio das cotações dos ativos de maior } \\
\text { negociabilidade dos setores de consumo cíclico. }\end{array}$ \\
\hline IDIV & 11 Índice de dividendos & $\begin{array}{l}\text { Indicar o desempenho médio de ativos que se destacaram em termos } \\
\text { de remuneração de investimento. }\end{array}$ \\
\hline IEE & $\begin{array}{l}10 \text { Índice de energia } \\
\text { elétrica }\end{array}$ & $\begin{array}{l}\text { Indicar o desempenho médio das cotações dos ativos de maior } \\
\text { negociabilidade do setor de energia elétrica. }\end{array}$ \\
\hline IFNC & 9 Índice financeiro & $\begin{array}{l}\text { Indicar o desempenho médio das cotações dos ativos de maior } \\
\text { representatividade dos setores intermediário financeiro. }\end{array}$ \\
\hline IGC & $\begin{array}{l}8 \text { Índice de governança } \\
\text { corporativa }\end{array}$ & $\begin{array}{l}\text { Indicar o desempenho médio das cotações dos ativos de empresas } \\
\text { listadas no novo mercado. }\end{array}$ \\
\hline IGCT & $\begin{array}{l}7 \text { Índice de governança } \\
\text { corporativa trade }\end{array}$ & $\begin{array}{l}\text { Indicar o desempenho médio das cotações dos ativos de emissão de } \\
\text { empresas integrantes do IGC. }\end{array}$ \\
\hline IMOB & 6 Índice imobiliário & $\begin{array}{l}\text { Indicar o desempenho médio das cotações de ativos com maior } \\
\text { negociabilidade dos setores de atividade imobiliária. }\end{array}$ \\
\hline
\end{tabular}




\begin{tabular}{|c|c|c|}
\hline Abreviação & Índice & Objetivo \\
\hline ITAG & $\begin{array}{l}5 \text { Índice de ações com } \\
\text { tag along diferenciado }\end{array}$ & $\begin{array}{l}\text { Indicar o desempenho médio dos ativos de empresas que oferecem } \\
\text { melhores condições aos acionistas minoritários, no caso de alienação de } \\
\text { controle. }\end{array}$ \\
\hline IVBX & $\begin{array}{l}4 \text { Índice valor Brasil, } \\
\text { Bolsa, Balcão }\end{array}$ & $\begin{array}{l}\text { Indicar o desempenho médio das cotações dos } 50 \text { ativos relacionados } \\
\text { em ordem decrescente por liquidez. }\end{array}$ \\
\hline MLCX & 3 Índice midLarge Cap & $\begin{array}{l}\text { Indicar o desempenho médio das cotações dos ativos de uma carteira } \\
\text { composta pelas empresas de maior capitalização. }\end{array}$ \\
\hline SMLL & 2 Índice small cap & $\begin{array}{l}\text { Indicar o desempenho médio das cotações dos ativos de uma carteira } \\
\text { composta pelas empresas de menor capitalização. }\end{array}$ \\
\hline UTIL & $\begin{array}{l}1 \text { Índice de utilidade } \\
\text { pública }\end{array}$ & $\begin{array}{l}\text { Indicar o desempenho médio das cotações dos ativos de maior } \\
\text { negociabilidade e representatividade do setor de utilidade pública } \\
\text { (energia elétrica, água e saneamento e gás). }\end{array}$ \\
\hline
\end{tabular}

Fonte: Adaptada de "Relate ou explique," por Brasil, Bolsa, Balcão, 2019.

Assim, esta pesquisa é quantitativa, pois foi realizada por meio de análise multivariada. Além dos testes de hipóteses, foram realizadas correlação e regressão múltipla.

\section{ANÁLISE E DISCUSSÃO}

Nesta seção são abordados as estatísticas descritivas, os testes de normalidades até a correlação de Pearson e os testes de hipóteses de t de Student, e ainda, regressão linear múltipla.

\subsection{ANÁLISE DESCRITIVA}

Os dados observados totalizam uma amostra de 1.238, entre os índices Amplos, de Governança, Segmentos, Sustentabilidade e Setoriais. Nos índices Amplos, o Índice Bovespa teve a maior variação, pois o valor mínimo foi de $-4,87$ e o máximo de 6,60. Entre os índices amplos, a maior média no período em questão é do índice Brasil Amplo - IBRX, com média de 0,0280, seguido dos índices IBRX e IBXL, com 0,0277 e 0,0258, respectivamente. Entre estes, o IBOV é o que detém o menor valor, de 0,0155. A Tabela 2 demonstra as estatísticas descritivas:

Tabela 2

Estatística descritiva dos indicadores estudados

\begin{tabular}{lccrrr}
\hline \multicolumn{1}{c}{ Índices } & N & Mínimo & Máximo & Média & Desvio padrão \\
\hline IBOV & 1238 & $-4,87$ & 6,60 &, 0155 & 1,48434 \\
IBRA & 1238 & $-4,51$ & 6,14 &, 0280 & 1,32975 \\
IBRX 100 & 1238 & $-4,56$ & 6,16 &, 0277 & 1,35066 \\
IBRX 50 & 1238 & $-4,77$ & 6,45 &, 0258 & 1,41948 \\
\hline
\end{tabular}




\begin{tabular}{|c|c|c|c|c|c|}
\hline Índices & $\mathrm{N}$ & Mínimo & Máximo & Média & Desvio padrão \\
\hline \multicolumn{6}{|c|}{ Índices de Governança } \\
\hline IGCX & 1238 & $-4,05$ & 5,44 & 0313 & 1,17645 \\
\hline IGCT & 1238 & $-4,59$ & 6,23 & 0312 & 1,27454 \\
\hline ITAG & 1238 & $-4,34$ & 6,36 & 0394 & 1,24270 \\
\hline \multicolumn{6}{|c|}{ Índices de Segmentos } \\
\hline IDIV & 1238 & $-5,24$ & 6,31 & ,0195 & 1,48149 \\
\hline MLCX & 1238 & $-4,76$ & 6,39 & 0314 & 1,36977 \\
\hline SMLL & 1238 & $-4,18$ & 4,21 &, 0002 & 1,13371 \\
\hline IVBX & 1238 & $-4,27$ & 4,78 & 0392 & 1,12081 \\
\hline \multicolumn{6}{|c|}{ Índices de Sustentabilidade } \\
\hline ISE & 1238 & $-4,54$ & 4,67 & 0211 & 1,16041 \\
\hline ICO2 & 1238 & $-4,57$ & 6,42 & 0353 & 1,30048 \\
\hline \multicolumn{6}{|c|}{ Índices Setoriais } \\
\hline IFNC & 1238 & $-6,04$ & 9,75 & ,0638 & 1,63474 \\
\hline IMOB & 1238 & $-5,30$ & 5,81 &,- 0089 & 1,56592 \\
\hline UTIL & 1238 & $-10,45$ & 5,01 & ,0300 & 1,39829 \\
\hline IEE & 1238 & $-8,17$ & 5,12 & 0169 & 1,31419 \\
\hline ICON & 1238 & $-4,39$ & 5,34 & ,0474 & 1,08865 \\
\hline
\end{tabular}

Entre os índices de governança, destaca-se o retorno médio diário do Índice de Tag Along, com o valor de 0,0394. Já o Índice de Governança Corporativa Diferenciada e o Índice de Governança Corporativa Trade tem a média muito próxima, 0,0313 e 0,0312, respectivamente. Em comparação com os demais índices, os de governança são os que detêm o valor médio mais alto, pois, em média, os retornos diários desses índices são de $3,40 \%$. Os índices setoriais detêm a segunda maior média, com 2,98\%; os de sustentabilidade com $2,82 \%$, em terceiro; e os índices amplos em quarto, com 2,42\%. Assim, os de segmentos detêm o menor valor médio, com $2,26 \%$, no período analisado.

Nos índices de segmentos o Small Caps deteve o menor retorno diário, com valor de 0,0002. Quanto ao Índice de Sustentabilidade, o retorno médio diário foi de 0,0211, menor em comparação ao Índice de Carbono Eficiente (ICO2). Todavia, o Índice Imobiliário, com o valor médio diário de -0,0089, é o índice com o menor retorno de todos os analisados. Para o desvio padrão, o Índice Financeiro detém o maior valor, de 1,63474. Em contrapartida, com 1,08865, o Índice de Consumo (ICON) detém o menor desvio padrão.

\subsection{NORMALIDADE DA AMOSTRA}

Para identificar se os testes estatísticos são adequados para a amostra, referente aos cinco anos de cada índice, foi realizado o teste de normalidade de Kolmogorov-Smirnov K-S e o teste Shapiro-Wilk. A Tabela 3 demonstra os índices e a significância destes nos respectivos testes: 
Tabela 3

Testes de normalidade Kolmogorov-Smirnov e Shapiro-Wilk

\begin{tabular}{|c|c|c|c|c|c|c|}
\hline \multirow{2}{*}{ Índices } & \multicolumn{3}{|c|}{ Kolmogorov-Smirnov ${ }^{a}$} & \multicolumn{3}{|c|}{ Shapiro-Wilk } \\
\hline & Estatística & $d f$ & Sig. & Estatística & $d f$ & Sig. \\
\hline \multicolumn{7}{|c|}{ Índices Amplos } \\
\hline IBOV &, 032 & 1238 &, 004 & 994 & 1238 &, 000 \\
\hline IBRA & ,046 & 1238 &, 000 & 991 & 1238 &, 000 \\
\hline IBRX 100 &, 044 & 1238 &, 000 & 991 & 1238 &, 000 \\
\hline IBRX 50 & 039 & 1238 &, 000 & 992 & 1238 &, 000 \\
\hline \multicolumn{7}{|c|}{ Índices de Governança } \\
\hline IGC &, 035 & 1238 & ,001 & ,990 & 1238 &, 000 \\
\hline IGCT &, 039 & 1238 & ,000 & 990 & 1238 &, 000 \\
\hline ITAG &, 043 & 1238 &, 000 & 987 & 1238 &, 000 \\
\hline \multicolumn{7}{|c|}{ Índices de Segmentos } \\
\hline IDIV & ,050 & 1238 & ,000 & 987 & 1238 &, 000 \\
\hline MLCX & 039 & 1238 & ,000 & 991 & 1238 &, 000 \\
\hline SMLL & , 049 & 1238 &, 000 & 990 & 1238 &, 000 \\
\hline IVBX & 036 & 1238 & ,001 & ,993 & 1238 &, 000 \\
\hline \multicolumn{7}{|c|}{ Índices de Sustentabilidade } \\
\hline ISE &, 032 & 1238 &, 005 & 993 & 1238 &, 000 \\
\hline ICO2 & 035 & 1238 & ,001 & 989 & 1238 &, 000 \\
\hline \multicolumn{7}{|c|}{ Índices Setoriais } \\
\hline ICON &, 032 & 1238 &, 005 & 991 & 1238 &, 000 \\
\hline IEE & 050 & 1238 & ,000 & 978 & 1238 &, 000 \\
\hline IFNC &, 047 & 1238 & ,000 & 980 & 1238 &, 000 \\
\hline IMOB & 038 & 1238 & ,000 & 993 & 1238 &, 000 \\
\hline UTIL & 051 & 1238 &, 000 & 973 & 1238 &, 000 \\
\hline
\end{tabular}

No teste de Kolmogorov-Smirnov todos os valores de significância ficaram abaixo de $5 \%$. Logo, rejeita-se a hipótese nula de que os dados apresentam normalidade. Dessa forma, tanto os índices amplos quanto os índices de governança, segmentos, sustentabilidade e setoriais apresentaram não normalidade. E ainda, no teste de Shapiro-Wilk, também se obtiveram valores abaixo de $5 \%$. Portanto, rejeita-se a hipótese de que os índices apresentam uma distribuição normal.

Entretanto, visto que os índices detêm retornos médios, variações mínimas, máximas e desvios padrões divergentes dos valores encontrados para os índices de sustentabilidade, é pertinente dar ênfase ao Índice de Sustentabilidade. A correlação entre os demais índices e o ISE pode demonstrar que mesmo com retornos diferentes, o retorno médio das empresas compostas no ISE terá a mesma direção, seja esta positiva, seja negativa. Assim, por meio da correlação, é possível demonstrar se qualquer dos segmentos é inversamente correlacionado com as práticas exigidas de sustentabilidade. 


\subsection{CORRELAÇÃO ENTRE ISE E OS DEMAIS ÍNDICES}

Por meio da correlação de Pearson, entre a variação dos retornos médio diários do ISE e os demais índices da Brasil, Bolsa, Balcão é possível analisar a direção dessa correlação e, com isso, se os retornos das empresas de determinados índices refletem de forma negativa ou positiva o índice de sustentabilidade. Assim, temos as seguintes hipóteses:

$\mathrm{H}_{\mathrm{o}} \mathrm{O}$ retorno médio dos índices da Brasil, Bolsa, Balcão está positivamente correlacionado com o ISE.

$\mathrm{H}_{1:} \mathrm{O}$ retorno médio dos índices da Brasil, Bolsa, Balcão não está positivamente correlacionado com o ISE.

Na Tabela 4 demonstram-se as correlações de Pearson entre o ISE e os demais índices da Brasil, Bolsa, Balcão para responder às hipóteses propostas neste estudo:

Tabela 4

Correlação de Pearson entre o ISE e os demais indicadores

\begin{tabular}{|c|c|c|}
\hline Índice & Coeficiente correlação com o ISE & Sig. ( 2 extremidades) \\
\hline \multicolumn{3}{|c|}{ Índices Amplos } \\
\hline IBOV &, $925^{* *}$ & ,000 \\
\hline IBRA &, $943^{* *}$ & ,000 \\
\hline IBRX 100 &, $943^{* *}$ & ,000 \\
\hline IBRX 50 & $935^{* *}$ &, 000 \\
\hline \multicolumn{3}{|c|}{ Índices de Governança } \\
\hline IGCX &, $954^{* *}$ & ,000 \\
\hline IGCT &, $954^{* *}$ & ,000 \\
\hline ITAG &, $928^{* *}$ &, 000 \\
\hline \multicolumn{3}{|c|}{ Índices de Segmentos } \\
\hline IDIV & $897^{* *}$ &, 000 \\
\hline MLCX &, $941^{* *}$ &, 000 \\
\hline SMLL &, $832^{* *}$ &, 000 \\
\hline IVBX &, $934^{* *}$ &, 000 \\
\hline \multicolumn{3}{|c|}{ Índices de Sustentabilidade } \\
\hline $\mathrm{ICO} 2$ & $942^{* *}$ &, 000 \\
\hline \multicolumn{3}{|c|}{ Índices Setoriais } \\
\hline ICON &, $833^{* *}$ & , 000 \\
\hline IEE &, $738^{* *}$ &, 000 \\
\hline IFNC &, $846^{* *}$ &, 000 \\
\hline IMOB & ,727** & ,000 \\
\hline UTIL &, $746^{* *}$ &, 000 \\
\hline
\end{tabular}


Assim, não se rejeita $\mathrm{H}_{0^{\prime}}$ pois o retorno médio dos índices da Brasil, Bolsa, Balcão está positivamente correlacionado com o ISE.

Com relação aos índices amplos, a correlação encontrada demonstrou-se forte e de forma positiva, e ainda, significante ao nível de 1\%. Mesmo que detenha retorno, variações e desvio padrão diferentes, o Índice de Sustentabilidade também reflete as variações dos índices amplos: IBOV, IBRA, IBRX 100 e IBRX 50. Logo, as empresas que compõem os índices amplos serão afetadas pelo desempenho do índice de sustentabilidade e vice-versa.

Também é válido para a correlação entre os índices de governanças (IGCX, IGCT e ITAG) que obtiveram os valores mais fortes correlacionados aos demais índices. Em média, a correlação foi de 0,94 , concomitantemente com os índices amplos também deteve correlação forte, em média, com 0,94.

A correlação mais fraca encontrada foi entre o ISE e o índice dos setores da atividade imobiliária (IMOB), de 0,746. Entretanto, a correlação existente entre ambos também foi positiva e forte.

A maior correlação existente no estudo foi a do ISE com o Índice de Governança Trade e Diferenciada, de 0,954.

Nota-se que todos os índices tiveram retornos fortemente correlacionados e de forma positiva. Esse resultado responde à pergunta desta pesquisa: o ISE reflete os demais índices listados anteriormente. Na próxima subseção está descrita a regressão linear múltipla.

\subsection{INFLUÊNCIA DOS DEMAIS ÍNDICES NO ISE}

Em razão de os índices compartilharem alguns ativos, mesmo com a ponderação da participação percentual diferente, o ISE pode ser explicado pela variação dos demais. Assim, considerando o efeito da correlação, este estudo chega às seguintes hipóteses:

$\mathrm{H}_{\mathrm{o}}$ Os demais índices da Brasil, Bolsa, Balcão influenciam positivamente o ISE.

$H_{1:}$ Os demais índices da Brasil, Bolsa, Balcão não influenciam positivamente o ISE.

Para testar essas hipóteses, foi realizada uma regressão linear múltipla, composta pela Equação 1:

$$
\begin{array}{rl}
I S E=\phi_{0}+\phi_{1} I & I b o v+\phi_{2} I b r a+\phi_{3} I b r x 100+\phi_{4} I b r x 50+\phi_{5} I g c x+\phi_{6} I g c t \\
& +\phi_{7} I t a g+\phi_{8} I \text { Iiv }+\phi_{9} \text { Ilcx }+\phi_{10} \text { Smll }+\phi_{11} I v b x+\phi_{12} I c o n \\
& +\phi_{13} I c o 2+\phi_{14} \text { Iee }+\phi_{15} I f n c+\phi_{16} I m o b+\phi_{17} \text { Util }+\varepsilon_{i}
\end{array}
$$


Nessa equação, o ISE é explicado pelos demais índices estudados (IBOV, IBRA, IBRX 100, IBRX50, IGCX, IGCT, ITAG, IDIV, MLCX, SMLL, IVBX, ICON, ICO2, IEE, IFNC, IMOB e UTIL) descritos na metodologia. $O$ símbolo $\phi O$ é a constante e o erro é $\varepsilon i$. O procedimento adotado para a regressão foi o stepwise em decorrência de o número de variáveis ser relativamente grande, e ainda para encontrar o modelo com o maior r2 (Fávero, Belfiore, Silva, \& Chan, 2009).

Na Tabela 5 demonstra-se o resumo dos modelos, com os respectivos $r 2$ e o teste de autocorrelação de Durbin-Watson.

Tabela 5

Resumo dos modelos encontrados

\begin{tabular}{|c|c|c|c|c|c|}
\hline Modelo & $\mathbf{R}$ & $\mathrm{R}$ quadrado & $\begin{array}{l}\mathrm{R} \text { quadrado } \\
\text { ajustado }\end{array}$ & $\begin{array}{l}\text { Erro padrão } \\
\text { da estimativa }\end{array}$ & $\begin{array}{l}\text { Durbin- } \\
\text { Watson }\end{array}$ \\
\hline IGCT & 954 & 911 & 911 & ,34658 & \\
\hline IGCT, IFNC & 964 & 930 & 930 & ,30784 & \\
\hline (3) IGCT, IFNC, IVBX & 966 & 934 & 934 & 29860 & \\
\hline (4) IGCT, IFNC, IVBX, IMOB & 969 & 939 & 939 & 28623 & \\
\hline (5) IGCT, IFNC, IVBX, IMOB, ICO2 & 970 & 941 & 941 & 28157 & \\
\hline $\begin{array}{l}\text { (6) IGCT, IFNC, IVBX, IMOB, ICO2, } \\
\text { ICON }\end{array}$ & 972 & 944 & 944 & ,27478 & \\
\hline $\begin{array}{l}\text { (7) IGCT, IFNC, IVBX, IMOB, ICO2, } \\
\text { ICON, IGCX }\end{array}$ & 973 & 946 & 946 & ,27033 & \\
\hline $\begin{array}{l}\text { (8) IGCT, IFNC, IVBX, IMOB, ICO2, } \\
\text { ICON, IGCX, IBOV }\end{array}$ & 973 & 947 & 947 & 26743 & \\
\hline $\begin{array}{l}\text { (9) IGCT, IFNC, IVBX, IMOB, ICO2, } \\
\text { ICON, IGCX, IBOV, UTIL }\end{array}$ & 974 & 948 & 948 & , 26532 & 2,034 \\
\hline
\end{tabular}

Dos 16 índices estudados, foram encontrados 9 modelos, sendo que o maior $r^{2}$ foi de 0,948 . Com o valor próximo a 2 , no teste de Durbin-Watson não existiu autocorrelação ao nível de significância de $5 \%$. Dessa forma, o modelo que mais influenciou as variações do ISE foi o de 9, o qual é composto pelas seguintes variáveis: Índice de Governança Trade, IGCT, Índice Financeiro, Índice Valor (Brasil, Bolsa, Balcão), Índice Imobiliário, Índice de Carbono Eficiente, Índice de Governança Corporativa Diferenciada, Índice Bovespa e Índice de Utilidade Pública.

Quanto à adequação do modelo encontrado, na Tabela 6 demonstram-se a análise da variância e o $p$ valor do teste f para o modelo 9 : 
Tabela 6

Análise da variância

\begin{tabular}{|c|c|c|c|c|c|c|}
\hline & Modelo & $\begin{array}{l}\text { Soma dos } \\
\text { Quadrados }\end{array}$ & $d f$ & $\begin{array}{l}\text { Quadrado } \\
\text { Médio }\end{array}$ & $F$ & Sig. \\
\hline \multirow{3}{*}{9} & Regressão & 1579,230 & 9 & 175,470 & 2492,662 &, $000^{i}$ \\
\hline & Resíduos & 86,445 & 1228 & , 070 & & \\
\hline & Total & 1665,674 & 1237 & & & \\
\hline
\end{tabular}

O modelo 9, encontrado pela regressão realizada por meio do procedimento stepwise, demonstrou-se altamente significante, pois o $p$ valor estabelecido no teste $f$ demonstrou-se ao nível de $1 \%$, sendo que ao menos uma das variáveis é estatisticamente diferente de zero. Assim, o modelo foi adequado para explicar a variação do ISE.

Ao nível de significância de $1 \%$, as variáveis compostas no modelo 9 foram estatisticamente diferentes de zero, conforme o $\mathrm{p}$ valor do teste $t$. Na Tabela 7 demonstramse os coeficientes encontrados para estimar a equação e a significância por meio do teste $t$ de Student.

Tabela 7

Coeficientes, teste te multicolinearidade

\begin{tabular}{|c|c|c|c|c|c|c|c|c|}
\hline & \multirow{2}{*}{$\begin{array}{c}\text { Modelo } \\
\text { B }\end{array}$} & \multicolumn{2}{|c|}{$\begin{array}{c}\text { Coeficientes não } \\
\text { padronizados }\end{array}$} & \multirow[t]{2}{*}{$\begin{array}{l}\text { Coeficientes } \\
\text { padronizados }\end{array}$} & \multirow{2}{*}{$t$} & \multirow{2}{*}{$\begin{array}{c}\text { Sig. } \\
\text { Tolerância }\end{array}$} & \multicolumn{2}{|c|}{$\begin{array}{l}\text { Estatísticas de } \\
\text { colinearidade }\end{array}$} \\
\hline & & $\begin{array}{l}\text { Modelo } \\
\text { padrão }\end{array}$ & Beta & & & & VIF & \\
\hline & (Constante) &,- 005 &, 008 & & -655 &, 513 & & \\
\hline & $\phi_{1} I g c t$ & ,306 & 091 & ,336 & 3,363 & ,001 & ,004 & 236,435 \\
\hline & $\phi_{2} I f n c$ &,- 321 & 019 &,- 452 & $-16,569$ & ,000 & , 057 & 17,604 \\
\hline & $\phi_{3} I v b x$ & 223 &, 032 & ,216 & 6,981 & ,000 &, 044 & 22,551 \\
\hline & $\phi_{4} I m o b$ & -091 & ,009 & -122 & $-9,968$ & ,000 & 281 & 3,561 \\
\hline$y$ & $\phi_{5} I c o 2$ &, 515 & ,043 & ,578 & 12,036 & ,000 & ,018 & 54,515 \\
\hline & $\phi_{6}$ Icon &,- 214 & ,021 &,- 200 & $-10,065$ & ,000 & 107 & 9,390 \\
\hline & $\phi_{7} \operatorname{Igcx}$ & ,634 & 105 & ,643 & 6,018 & ,000 & ,004 & 270,068 \\
\hline & $\phi_{8} I b o v$ & 109 & ,023 & 140 & $-4,746$ & ,000 & ,049 & 20,474 \\
\hline & $\phi_{9} U t i l$ & , 040 & ,009 & ,048 & 4,541 & ,000 & ,376 & 2,656 \\
\hline
\end{tabular}

Todavia, vale ressaltar a presença de multicolinearidade nas variáveis em que o valor da Variance Inflation Factor (VIF) foi superior a 10. Entretanto, não afetou a intenção de previsão, pois os mesmos padrões de relação entre as variáveis explicativas também foram incorporados. Assim, a solução para a multicolinearidade se limitou apenas em identificá-la (Gujarati \& Porter, 2011). 
Dessa forma, notou-se que os índices IGCT, IVBX, ICO2, IGCX e UTIL obtiveram o valor esperado: positivo. Dessa forma, a $\mathrm{H}_{0}$ para esses índices foi confirmada. Entretanto, os valores para os índices IFNC, IMOB, ICON e IBOV influenciaram de forma negativa o Índice de Sustentabilidade Ambiental, rejeitando-se $\mathrm{H}_{\mathrm{o}}$ para esses índices.

\section{CONSIDERAÇÕES FINAIS}

O presente trabalho objetivou analisar a relação e a influência dos vários índices da Brasil, Bolsa, Balcão, no ISE. Os resultados demonstraram que a relação do ISE com os demais índices é forte, positiva e estatisticamente significante. Portanto, esses resultados apontam que as empresas buscam se legitimar participando desse índice, isto é, buscam incorporar cada vez mais estruturas isomórficas que refletem o ambiente institucional. $\mathrm{E}$ assim, a adoção do ISE pode ser apenas mais um rótulo.

Com isso, questiona-se a legitimidade desse Índice, pois, se existe relação com todos os indicadores, basta às empresas se suprimirem às regras dos índices que são pertinentes aos seus interesses.

Por outro lado, a regressão realizada por meio do método stepwise encontrou uma equação com $r^{2}$ 0,948, e ainda, com o teste $f$, demonstrou-se a significância de $1 \%$. Logo, rejeitou-se a hipótese de as variáveis dos modelos serem estatisticamente iguais a zero. Assim, as principais influências positivas no ISE foram estabelecidas pelos índices IGCT, IVBX, ICO2, IGCX e UTIL, ao nível de significância de 1\%. Já os índices IFNC, IMOB, ICON e IBOV influenciaram de forma negativa, com ressalva da presença de multicolinearidade, em que os valores Variance Inflation Factor (VIF) são superiores a 10.

Dessa forma, é possível identificar que o ISE foi influenciado de forma significante por 9 índices dos 17 comparados, sendo que 5 deles influenciaram de forma positiva e 4 de forma negativa. Logo, a legitimidade proporcionada pelo ISE pode ser atrelada à composição dos demais índices que o explicam.

O conceito de sustentabilidade representa uma nova abordagem de se fazer negócios (Silva et al., 2009) e é assumido por um novo nicho de investidores, mas não reflete os resultados desta pesquisa em razão de a variação do ISE ser explicada e correlacionada com os demais indicadores.

Contudo, tem-se como limitação de pesquisa a utilização apenas de variáveis referentes aos índices da Brasil, Bolsa, Balcão. Muitas das empresas, mesmo que com ponderação diferente, têm os seus ativos ponderando mais de um índice. Como sugestão para futuras pesquisas, apresenta-se abordar a correlação e a influência dos indicadores de desempenho das empresas, como $\mathrm{Q}$ de Tobin, ROA, ROE e ROI com os principais índices, o que pode 
demonstrar quais são pertinentes para as empresas se sujeitarem às suas prescrições e não atenderem apenas ao isomorfismo do mercado.

\section{REFERÊNCIAS}

Agustia, D., Sawarjuwono, T., \& Dianawati, W. (2019). The Mediating Effect of Environmental Management Accounting on Green Innovation-Firm Value Relationship. International Journal of Energy Economics and Policy, 9(2), 299-306.

Brandão, H. P., \& Guimarães, T. D. A. (2001). Gestão de competências e gestão de desempenho: Tecnologias distintas ou instrumentos de um mesmo construto? Revista de Administração de Empresas, 4l(1), 8-15.

Brasil, Bolsa, Balcão. (2017). Metodologia. Recuperado de https://www.isebvmf.com.br

Brasil, Bolsa, Balcão. (2019). Relate ou explique. Recuperado de http://www.b3.com.br/pt_ $\mathrm{br} / \mathrm{b} 3 /$ sustentabilidade/nas-empresas/

Cristófalo, R. G., Akaki, A. S., Abe, T. C., Morano, R. S., \& Miraglia, S. G. E. K. (2016). Sustentabilidade e o mercado financeiro: Estudo do desempenho de empresas que compõem o índice de sustentabilidade empresarial (ISE). REGE-Revista de Gestão, 23(4), 286-297.

Deliberal, J. P., Tondolo, V. A. G., Camargo, M. E., \& Tondolo, R. D. R. P. (2016). Environmental management as a strategic capability: A study on the furniture manufacturing cluster of Southern Brazil. Brazilian Business Review, 13(4), 118-140.

Favaro, L. C., \& Rover, S. (2014). Índice de Sustentabilidade Empresarial (ISE): A associação entre os indicadores econômico-financeiros e as empresas que compõem a carteira. Contabilometria, $1(1)$.

Fávero, L. P., Belfiore, P., Silva, F. D., \& Chan, B. L. (2009). Análise de dados-Modelagem multivariada para tomada de decisões. Rio de Janeiro: Campus.

Fundação Getulio Vargas-Escola de Administração de Empresas de São Paulo. (2017). ISE BM\&FBovespa. Recuperado de http://grces.com.br/ise-bm-fbovespa

Furtado, L. L., Antonovz, T., Correa, M. D., Silva, O. A. D. P. da, \& Panhoca, L. (2019). Relação entre Sustentabilidade e Inovação: Uma análise da legitimidade organizacional das empresas do setor elétrico brasileiro. Revista Catarinense da Ciência Contábil, 18, $1-16$. 
Gomes, A. R. V. (2018). Governança corporativa e seus determinantes: Um estudo com a qualidade da informação contábil, competitividade de mercado e risco não sistêmico (Dissertação de mestrado). Universidade Estadual do Oeste do Paraná, Cascavel. Recuperado de $\mathrm{http}: / /$ tede.unioeste.br/handle/tede/3806

Guimarães, T. M., Peixoto, F. M., \& Carvalho, L. (2017). Sustentabilidade empresarial e governança corporativa: Uma analise da relação do ISE da BM\&FBOVESPA com a Compensação dos Gestores de Empresas Brasileiras. Revista de Educação e Pesquisa em Contabilidade, 11(2), 134-149.

Gujarati, D. N., \& Porter, D. C. (2011). Econometria Básica. (5ª ed.). Poro Alegre: AMGH.

Hair, J. F., Jr., Babin, B., Money, A. H., \& Samouel, P. (2005). Fundamentos de métodos de pesquisa em administração. Porto Alegre: Bookman.

Hart, S. L., \& Ahuja, G. (1996). Does it pay to be green? An empirical examination of the relationship between emission reduction and firm performance. Business strategy and the Environment, 5(1), 30-37.

Henri, J. F., \& Journeault, M. (2010). Eco-control: The influence of management control systems on environmental and economic performance. Accounting, Organizations and Society, 35(1), 63-80.

Hermawan, A., Aisyah, I. S., Gunardi, A., \& Putri, W. Y. (2018). Going green: Determinants of carbon emission disclosure in manufacturing companies in Indonesia. International Journal of Energy Economics and Policy, 8(1), 55-61.

Lima, S. L. L. (2018). Fatores que influenciam a probabilidade das práticas de disclosure de informações financeiras das empresas (Dissertação de mestrado). Universidade Estadual do Oeste do Paraná, Cascavel. Recuperado de http://tede.unioeste.br/handle/ tede/3665

Lima, S. L. L., Gomes, A. R. V., Priebe, A. C., Dall'Asta, D., \& Mello, G. R. (2018). Competitividade, governança e relevância da informação contábil: Uma análise do mercado brasileiro versus o norte-americano. Revista de Contabilidade, Ciência da Gestão e Finanças, $6(1), 37-61$.

Lindblom, C. K. (1994). The implications of organizational legitimacy for corporate social performance and disclosure. Paper presented a the Critical Perspectives on Accounting Conference, New York.

Macedo, F., Barbosa, H., Callegari, I., Monzoni, M., \& Simonetti, R. (2012). O Valor do ISE: Principais estudos e a perspectiva dos investidores. São Paulo: BM\&FBovespa. 
Meyer, J. W., \& Rowan, B. (1977). Institutionalized organizations: Formal structure as myth and ceremony. American Journal of Sociology, 83(2), 340-363.

Nascimento, S., Rocha, I., Kroenke, A., Silva, J. O., \& Hein, N. (2012). Avaliação de desempenho organizacional: Ferramentas gerenciais utilizadas em pesquisas nacionais da área de engenharia. P\&D em Engenharia de Produção, 10(1), 11-21.

O'Donovan, G. (2002). Environmental disclosures in the annual report: Extending the applicability and predictive power of legitimacy theory. Accounting, Auditing \& Accountability Journal, 15(3), 344-371. doi: 10.1108/09513570210435870

Oro, I. M., Balbinot, I. J., Thomé, D., \& Lavarda, C. E. F. (2014). Relação do capital intelectual de natureza social e ambiental com o desempenho econômicofinanceiro do segmento de energia elétrica brasileiro. Revista de Administração da Universidade Federal de Santa Maria, 7, 59-75.

Parsons, T. (1956). Suggestions for a sociological approach to the theory of organizations-I. Administrative Science Quarterly, 1(1), 63-85.

Pascuotte, D. (2012). Efeito no preço e volume das ações das companhias ingressantes no Índice de Sustentabilidade Empresarial (ISE). Revista de Finanças Aplicadas, 1, 13.

Sales, B., Rover, S., \& Ferreira, J. S. (2018). Coerência na evidenciação das práticas ambientais das empresas listadas no índice de sustentabilidade empresarial (ISE). Revista Ambiente Contábil, 10(2), 1-22.

Silva, J. O. D., Rocha, I., Wienhage, P., \& Rausch, R. B. (2009). Gestão ambiental: Uma análise da evidenciação das empresas que compõem o Índice de Sustentabilidade Empresarial (ISE). Revista de Gestão Social e Ambiental, 3(3).

Sonnentag, S., \& Frese, M. (2002). Performance concepts and performance theory. Psychological management of individual performance, 23(1), 3-25.

Suchman, M. C. (1995). Managing legitimacy: Strategic and institutional approaches. The academy of management review, 20(3), 571-600.

Venkatraman, N., \& Ramanujam, V. (1986). Measurement of business performance in strategy research: A comparison of approaches. Academy of management review, 11(4), 801814. 


\section{Como citar este artigo:}

\section{ABNT}

SOUZA, Roberto Francisco de et al. A legitimidade do Índice de Sustentabilidade Empresarial (ISE) frente aos demais Índices B3. RACE, Revista de Administração, Contabilidade e Economia, Joaçaba: Editora Unoesc, v. 18, n. 3, p. 521-542, set./dez. 2019. Disponível em: http://editora.unoesc.edu.br/index.php/race. Acesso em: dia/mês/ano.

\section{APA}

Souza, R. F. de, Gomes, A. R. V., Lima, S. L. L. de, Santos, G. V. dos, \& Dal Vesco, D. G.

(2019). A legitimidade do Índice de Sustentabilidade Empresarial (ISE) frente aos demais Índices B3. RACE, Revista de Administração, Contabilidade e Economia, 18(3), 521542. Recuperado de http://editora.unoesc.edu.br/index.php/race 
